\title{
Preparation and Characterization of Clay-polymer Nanocomposite Having Covalently- bound poly(norbornenes) with Pendant Cholesterols
}

\author{
David Penuliar Penaloza Jr. ${ }^{a} \mathbb{C}^{-}$, Thomas Andrew Patrick Seery ${ }^{b, c}$ \\ ${ }^{a}$ Chemistry Department, College of Science, De La Salle University, Manila, Philippines \\ ${ }^{b}$ Chemistry Department, University of Connecticut, Connecticut, USA \\ 'Polymer Program, Institute of Materials Science, University of Connecticut, Connecticut, USA
}

Received: March 13, 2018; Revised: July 08, 2018; Accepted: November 22, 2018

\begin{abstract}
We have successfully prepared a clay-based polymer nanocomposite having grown cholesterol bearing poly(norbornene) brushes end-tethered from a naturally-occurring montmorillonite clay template. The synthesis of this hybrid material involved the treatment of the clay with first generation Grubbs catalyst, a ruthenium alkylidene, which induced a ring-opening metathesis and subsequent surface initiated polymerization of the cholesterol bearing monomer. This results in an inorganic/ organic hybrid functional nanomaterial where the inclusion of the clay particles in a liquid crystalline domain modifies the thermal transitions.
\end{abstract}

Keywords: surface initiated polymerization, nanocomposite, liquid crystalline polymer, tethered polymer.

\section{Introduction}

Cholesterol-bearing polymers belong to a class of polymeric system called liquid crystalline polymers (LCPs). LCPs exhibit remarkable properties such as high heat resistance, remarkable electrical tolerance and excellent chemical stability LCPs have found applications in opto-electronics, color information technology, and biotechnology ${ }^{1-3}$.

In the area of nanocomposites, one interesting synthetic approach to prepare such materials is through surface initiated polymerizations (the "grafting from" approach) to produce complex materials with well-defined structures ${ }^{4-8}$. The growth of polymers directly from the surface-immobilized initiators using controlled polymerization techniques such as atom transfer radical polymerization (ATRP) $)^{9-14}$, reversible addition-fragmentation transfer polymerization (RAFT) ${ }^{15-17}$ and ring opening metathesis polymerization (ROMP) ${ }^{18-21}$, among others, had become a powerful strategy for anchoring well-defined polymers to substrates.

The rationale for using surface initiated polymerizations is to control the density of surface grafting a priori as well as the chain length for a variety of polymers. The current study marks the beginning of explorations of functional nanocomposites where the inclusion of the particles in a liquid crystalline domain modifies the thermal transitions.

\section{Materials and Methods}

Silylation of the host silicate clays. A commercial organoclay, Cloisite15A, from the Southern Clay Products, Inc. was used as a surface polymerization template. The organoclay was vacuum-dried at $50{ }^{\circ} \mathrm{C}$ prior to silylation.

*email:david.penaloza.jr@dlsu.edu.ph
A silane coupling agent having a norbornene moiety, [(bicycloheptenyl)ethyl] dimethylchlorosilane, was used to functionalize the clay. A $5.0 \mathrm{~g}$ sample of the organoclay was placed in an air-free three-neck round bottom flask and flushed with nitrogen for few minutes. $150 \mathrm{~mL}$ of dry hexane was then added to the sample and the mixture was stirred under static nitrogen for 10 minutes.

Then, $5 \mathrm{~mL}$ of norbornene-chlorosilane was added to the flask and the mixture was refluxed under nitrogen for $48 \mathrm{~h}$. The final product (silylated organoclay) was then filtered off and repeatedly washed with hexane, before being dried at $60{ }^{\circ} \mathrm{C}$ for $24 \mathrm{~h}$ under vacuum. (Yield $=91 \%$ )

Synthesis of Cloisite15A/polyNBCh9 nanocomposite. Synthesis was done inside a glovebox. A $100 \mathrm{mg}$ Cloisite_sil was placed in a $20-\mathrm{mL}$ scintillation vial and was brought inside glovebox. Five $\mathrm{mL}$ of distilled THF (stabilized with TCP, $0.69 \mathrm{~g}$ TCP per $150 \mathrm{~mL}$ THF) was used to disperse the clay. Fifteen mg of Grubbs 1st generation catalyst was then added to the clay solution and stirred for 5 minutes. The clay solution was then washed with $15 \mathrm{~mL}$ hexane to get rid of unbound catalyst, sealed with parafilm wax and brought outside the glovebox for centrifugation. After centrifugation, clay solution was brought back inside the glovebox, and the supernatant was pipetted off. Then the clay was dispersed in $5 \mathrm{~mL}$ THF. Washing with hexane was done two more times. After the last wash, the clay was redispersed in $5 \mathrm{~mL}$ of THF.

In a separate $20 \mathrm{~mL}$ scintillation vial, $470 \mathrm{mg}$ of NBCh-9 monomer was placed. Saturated solution of this monomer was prepared by adding anhydrous THF stabilized with tricyclohexylphosphine (TCP) drop by drop until all monomers were dissolved. The prepared cholesterol-norbornene solution was then added to the solution of activated silica particles and stirred for one hour. The polymerization was terminated by 
adding $1 \mathrm{~mL}$ of ethyl vinyl ether (EVE). The nanocomposite material was precipitated in cold methanol. The precipitate was then recovered by filtration and was vacuum dried for 24 hours prior to analysis. $($ Yield $=85 \%$ )

\section{Instrumentation}

Fourier transform infrared (FTIR) spectroscopy. To monitor the growth of the polymer chains from the clay template, infrared spectroscopy was employed. Fourier transform infrared (FTIR) spectra of the samples in $\mathrm{KBr}$ pellets were collected using a Nicolet Magna-560 FTIR spectrometer.

Thermogravimetric analysis (TGA). Thermogravimetric analysis was utilized to determine the organic (polymer and modifier) and the inorganic (clay) components of the nanocomposites. Samples were placed in alumina pan and were heated up to $800^{\circ} \mathrm{C}$ under nitrogen atmosphere with a heating rate of $10^{\circ} \mathrm{C} / \mathrm{min}$. TGA analyses of the clay and clay-polymer nanocomposites were carried out on a TA Instruments SDT Q600 Simultaneous TGA/DSC.

Differential scanning calorimetry (DSC). Differential scanning calorimetric (DSC) measurement was utilized to determine the thermal transition properties of the prepared nanocomposite as compared to that of the polymer only. Samples were placed in alumina pan and were heated up from $-5{ }^{\circ} \mathrm{C}$ to $150{ }^{\circ} \mathrm{C}$ under nitrogen atmosphere with a heating rate of $10^{\circ} \mathrm{C} / \mathrm{min}$ at three heating/cooling cycles. DSC analyses of the nanocomposite and the bare polymer were carried out on a TA Instruments DSC Q-20.

\section{Results and Discussion}

\subsection{Preparation of clay-polyNBCh9 nanocomposite}

This work describes the preparation of hybrid materials composed of an outer layer of cholesterolbearing poly(norbornenes) covalently bound from an inorganic substrate. In our approach, the nanocomposite was prepared in the following manner: (1) the inorganic surface (Cloisite15A) was initially functionalized with norbornenyl initiator ligands via a silane coupling reaction; and then, (2) a ruthenium-based metal alkylidene catalyst (first generation Grubbs catalyst) was used to activate the surface-bound norbornenyl ROMP initiators; and a chain growth polymerization of the norbornene derivative was directly conducted from the inorganic surface resulting in the formation of surface-tethered chains containing cholesteric mesogens. A schematic diagram outlining the two-step process as described above is shown in Figure 1. The nanocomposite was prepared by the addition of the norbornenyl-bearing inorganic template activated by the first generation Grubbs catalyst to a solution of the norbornene derivative dissolved in tetrahydrofuran (THF). The THF solvent was spiked with tricyclohexylphosphine (TCP) to increase the stability of the metal alkylidene catalyst bound on the surface of the host silicate clay template. Also, the presence of the free TCP ligand in the solution slows down the polymerization rate of norbornene in THF. Jordi and Seery $(2005)^{19}$ observed that the bound ruthenium catalysts on the silica nanoparticles surfaces were found to polymerize norbornene too rapidly when THF was not added with TCP. The polymerization was terminated by the addition of ethyl vinyl ether (EVE).

\subsection{Characterization of clay-polyNBCh9 nanocomposite}

To confirm the growth of the poly(norbornene) chains bearing cholesterol moieties on the clay template, FTIR and TGA measurements of the clay template and the resulting nanocomposites were done.

FTIR spectroscopy. Figure 2 shows the FTIR spectra of (a) a poly(norbornene) chain having pendant cholesterol groups, (b) the clay template used for the SI-ROMP, silylated organoclay Cloisite15A and (c) the clay-polymer nanocomposite prepared from the SI-ROMP of norbornene monomers bearing a cholesterol moiety. The silylated Cloisite 15A has characteristic peaks at 1045,525 and $467 \mathrm{~cm}^{-1}$ originating from the Si-O group. Also, the clay has IR peaks at 2919 and $2852 \mathrm{~cm}^{-1}$, referring to the assymmetric and symmetric C-H stretching bands, respectively, associated with the alkyl chains of the modifier (Figure 2b). Meanwhile, the following IR peaks are observed in the cholesterol-bearing poly(norbornenes), polyNBCh9: $\left(=\mathrm{CH}_{2}\right), 3066 \mathrm{~cm}^{-1}$; (aliphatic $\mathrm{C}-\mathrm{H}), 2929-2852 \mathrm{~cm}^{-1} ;(\mathrm{C}=\mathrm{O}), 1734 \mathrm{~cm}^{-1}$; and $(\mathrm{C}-\mathrm{O}), 1173$ $\mathrm{cm}^{-1}$ (Figure 2a).

The presence of the peaks at $1734 \mathrm{~cm}^{-1}(\mathrm{C}=\mathrm{O})$, that is inherent to the carbonyl group of the poly(norbornene) derivative and the peaks at 1045,525 and $467 \mathrm{~cm}^{-1}$ originating from the Si-O group in the FTIR spectrum of the nanocomposite (Figure 2c) confirms the growth of the polymer chains from the host silicate clay.

Thermogravimetric analysis. We ran TGA experiments on the clay, the unfilled polyNBCh9 and the nanocomposite to compare mass loss. The purpose of the TGA was to determine the composition of the nanocomposite using TGA data obtained from the clay template and the polymer.

Figure 3 shows the TGA thermograms of the (a) clay template, Cloisiste15A_sil; (b) nanocomposite, Cloisite15ApolyNBCh9 and (c) an unfilled polymer prepared from the same norbornene derivative, polyNBCh9.

For the clay template (Figure 3a), the decomposition of the organic substance (modifiers in the clay that includes the alkyl ammonium and norbornene-bearing silane) takes place in the range $200-450{ }^{\circ} \mathrm{C}$ and the total mass loss after heating to $800^{\circ} \mathrm{C}$ is $41 \%$. Meanwhile, the unfilled polyNBCh9 (Figure $3 \mathrm{c}$ ) thermally degrades around $400^{\circ} \mathrm{C}$ and registers 


\section{Modifying clay template to covalently attach the initiating group}

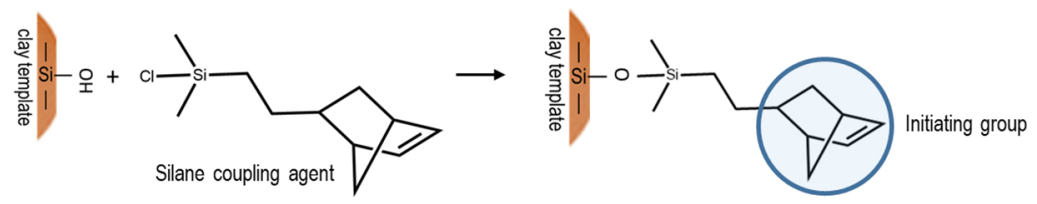

\section{End-tethered poly(norbornene) chains from the clay template via metal-mediated polymerization}
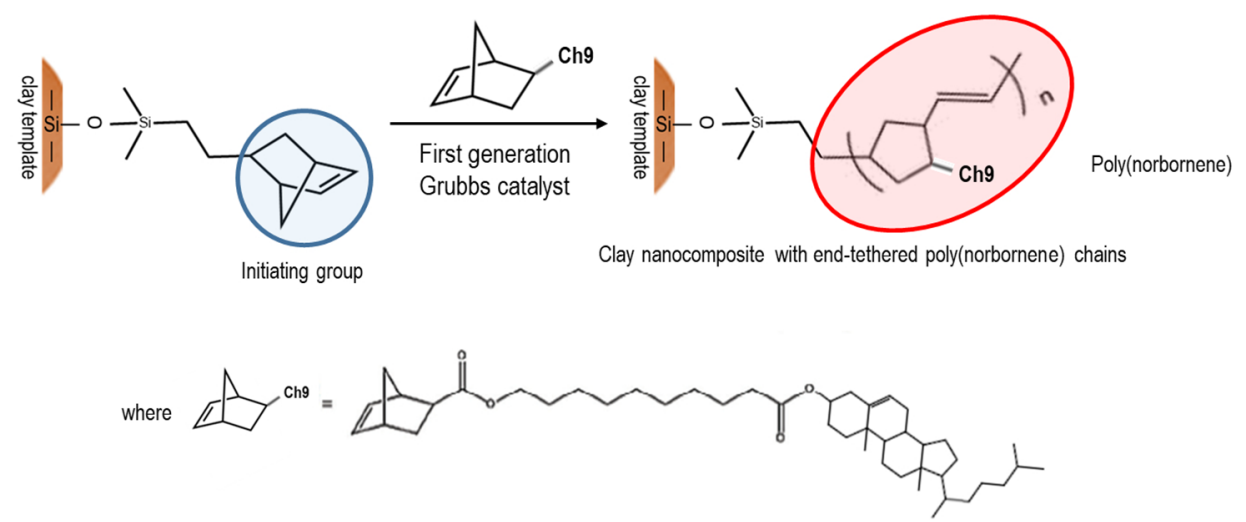

Figure 1. Schematic representation of the preparation of clay-based liquid crystalline polymer nanocomposite with end-tethered polymer having cholesterol pendant chains

$100 \%$ mass loss after heating at $800{ }^{\circ} \mathrm{C}$. Further, different decomposition steps can be identified. The decomposition profiles can be associated with the degradation of the cholesterol side chains and the subsequent degradation of the poly(norbornene) backbone as established by Ahn, Le and Kasi (2009) ${ }^{1}$ where they characterized different side-chain liquid crystalline polymers bearing cholesterol mesogens. To prepare these liquid crystalline polymers, a series of norbornene carboxylic cholesteryl ester monomers using various alkyl spacers were synthesized.

The nanocomposite exhibited a much higher mass loss relative to the clay template at $17 \%$ confirming the presence of the polymer in the nanocomposite. This result also complements the FTIR data. It is interesting to note however, that the nanocomposite degrades at a much lower temperature, around $310^{\circ} \mathrm{C}$ as compared to the unfilled polyNBCh9. In the studies involving nanocomposites, some papers indicated the decrease of thermal stability of polymer nanocomposites ${ }^{22-24}$ while other studies indicated otherwise ${ }^{25-27}$.

Differential scanning calorimetric (DSC) study of the homopolymer and the nanocomposite. All thermal transition temperatures of the polymer and the inorganic-polymer nanocomposite were determined using the 2 nd heating scans from $-50{ }^{\circ} \mathrm{C}$ to $150{ }^{\circ} \mathrm{C}$ at $10{ }^{\circ} \mathrm{C} / \mathrm{min}$. The glass transition temperature, $\mathrm{T}_{\mathrm{g}}$, of the homopolymer polyNBCh9 was determined at $40^{\circ} \mathrm{C}$ (Figure 4). Growing these chains from the MMT clay surfaces via surface initiated polymerization decreased the $\mathrm{T}_{\mathrm{g}}$ to $12^{\circ} \mathrm{C}$, respectively. It has been reported in the literature that the addition of nanofiller can change by as much as $\pm 30{ }^{\circ} \mathrm{C}$ of the $\mathrm{T}_{\mathrm{g}}$ of a polymer ${ }^{28-29}$. Bansal and co-researchers $(2006)^{29}$ found out the matrix polymers that wetted the nanoparticles showed an increase in glass transition temperature, and those that were non-wetting showed a decrease in glass transition temperature. Their study established the direct correlation between polymer wetting behavior and changes in the glass transition temperature. The study involved the use of poly(styrene) brushes having silica spheres. Further, in the work of Bandi and Schiraldi $(2006)^{30}$ where they studied the $T_{\mathrm{g}}$ behavior of clay aerogel/ poly(vinyl alcohol) composites, the change in $\mathrm{T}_{\mathrm{g}}$ is a result of two competing processes: (1) enhanced surface interaction resulting in a decrease in the chain mobility of the polymer architecture and (2) increased free volume at the interface due to lowered crystallinity of the chains, leading to increased chain mobility.

Aside from the glass transition, another thermal transition occurs at $88^{\circ} \mathrm{C}$ for the homopolymer, but is observed at a much lower temperature for the clay-polyNBCh9 nanocomposite, at $12{ }^{\circ} \mathrm{C}$. This temperature is where the side-chain crystallization occurs $\left(\mathrm{T}_{\mathrm{s}}\right)$. The observation of a side-chain crystallization for the homopolymer, polyNBCh9, can be attributed to the flexibility of the 9-carbon spacer connecting the cholesterol mesogens to the poly(norbornene) network ${ }^{1-2,31}$.

Another strong, sharp exothermic transition was also observed at $106^{\circ} \mathrm{C}$ for the polyNBCh9 referring to the clearing temperature, $\mathrm{T}_{\mathrm{cl}}$, the temperature at which the mesophase of the liquid crystalline polymer becomes isotropic. 


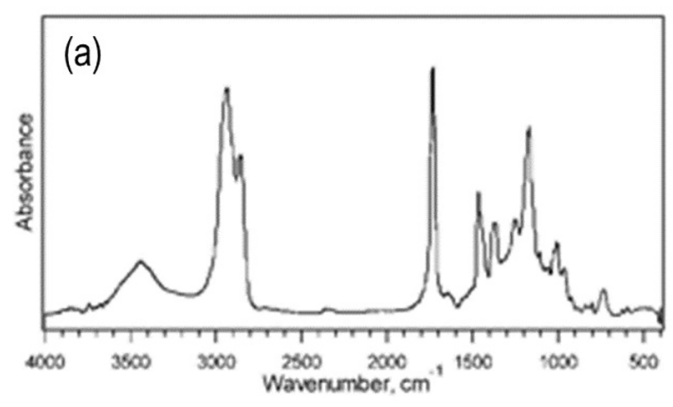

(b)
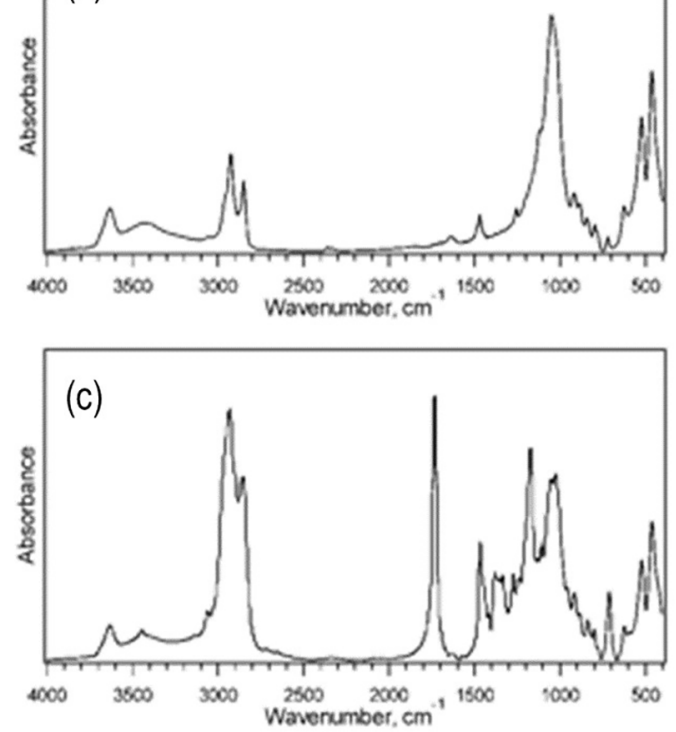

Figure 2. FTIR spectra of the (a) poly(norbornene) with cholesterolbearing pendant group, polyNBCh9, (b) silylated organomodified MMT clay, Cloisite15A(Cloisite15A_sil) and a (c) clay-polyNBCh9

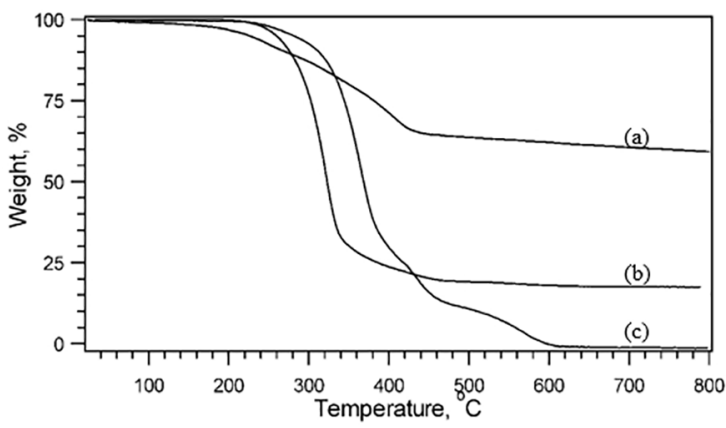

Figure 3. TGA thermograms of the (a) clay template (Cloisite15A_sil) and (b) a clay-polyNBCh9 nanocomposite obtained from the surfaceinitiated polymerization of NBCh9 monomers using Cloisite15A-sil clay as the template and (c) a poly(norbornene) with cholesterolbearing pendant groups, polyNBCh9

In summary, tethering the polymer containing cholesteric moieties changes the thermal properties of the prepared nanocomposites as compared to the bare (unfilled) homopolymer prepared from the same derivatized monomer.

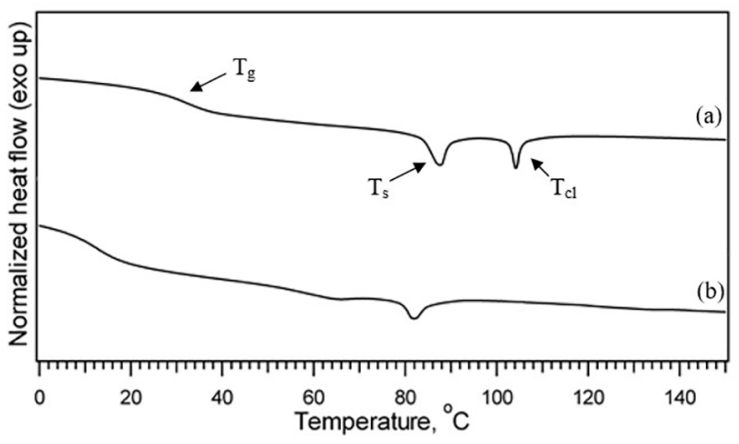

Figure 4. DSC thermograms $\left(10^{\circ} \mathrm{C} / \mathrm{min}\right.$, second heating scans $)$ of (a) homopolymer polyNBCh9 and (b) MMT-polyNBCh9. $\mathrm{T}_{\mathrm{g}}, \mathrm{T}_{\mathrm{s}}$ and $\mathrm{T}_{\mathrm{cl}}$ refer to the glass transition temperature, side-chain crystallization temperature and clearing temperature, respectively

\section{Conclusion}

We have successfully grown cholesterol-bearing poly(norbornene) brushes covalently attached from surfaces of silicate clays. The synthesis of these composites involved the treatment of inorganic host with first generation Grubbs catalyst, a ruthenium alkylidene, that induced a ring-opening metathesis and subsequent surface initiated polymerization of the cholesterol bearing monomer. Nanocomposites of this nature can serve as models for testing theories of polymer brushes in confined geometries. In the synthesis of these model systems, control over aspects of the polymer brushes such as brush density, molecular weight, and polydispersity is essential. Varying the organic moieties, i.e. the monomer used to grow the polymer chains, is one method of controlling these variables. Work is presently being done to further characterize the growth of these polymer chains formed in this fashion and their behavior as LCPs.

\section{Acknowledgments}

The authors would like to acknowledge Prof. Rajeswari Kasi (University of Connecticut) and Prof. Suk-kyun Ahn (University of Connecticut, now with Pusan National University, South Korea) for providing the derivatized norbornene containing cholesterol moieties used as the monomer for this work.

\section{References}

1. Ahn SK, Nguyen Le LT, Kasi RM. Synthesis and characterization of side-chain liquid crystalline polymers bearing cholesterol mesogen. Journal of Polymer Science Part A: Polymer Chemistry. 2009;47(10):2690-2701.

2. Trimmel G, Riegler S, Fuchs G, Slugove C, Stelzer F. Liquid Crystalline Polymers by Metathesis Polymerization. In: Buchmeiser MR, ed. Metathesis Polymerization. Berlin Heidelberg: Springer; 2005. p. 43-87. 
3. Pugh C, Kiste AL. Molecular engineering of side-chain liquid crystalline polymers by living polymerizations. Progress in Polymer Science. 1997;22(4):601-691.

4. Osicka J, Ilcíková M, Mrlik M, MinaríkA, Pavlinek V, Mosnácek J. The Impact of Polymer Grafting from a Graphene Oxide Surface on its Compatibility with a PDMS Matrix and the Light-Induced Actuation of the Composites. Polymers. 2017;9(7):264.

5. Xie MR, Dang JY, Shi JX, Han HJ, Ng C, Huang W, et al. Synthesis and self-assembly of well-defined polymer brushes with high grafting density of hydrophobic poly(e-caprolactone) and hydrophilic poly(2-(dimethylamino)ethyl methacrylate) side chains. Acta Chimica Sinica. 2009;67(8):869-874.

6. Advincula R. Polymer Brushes by Anionic and Cationic SurfaceInitiated Polymerization (SIP). In: Jordan R, ed. Surface-Initiated Polymerization I. Berlin: Springer-Verlag; 2006. p. 107-136.

7. Wu T, Efimenko K, Genzer J. Combinatorial Study of the Mushroomto-Brush Crossover in Surface Anchored Polyacrylamide. Journal of the American Chemical Society. 2002;124(32):9394-9395.

8. Zhao B, Brittain WJ. Polymer brushes: surface-immobilized macromolecules. Progress in Polymer Science. 2000;25(5):677710.

9. Börner HG, Beers K, Matyjaszewski K, Sheiko SS, Möller M. Synthesis of Molecular Brushes with Block Copolymer Side Chains Using Atom Transfer Radical Polymerization. Macromolecules. 2001;34(13):4375-4383.

10. Chen M, Qin L, Liu Y, Zhang F. Controllable preparation of polymer brushes from mesoporous silica SBA-15 via surfaceinitiated ARGET ATRP. Microporous and Mesoporous Materials. 2018;263:158-164.

11. Chmielarz P, Krys P, Wang Z, Wang Y, Matyjaszewski K. Synthesis of Well-Defined Polymer Brushes from Silicon Wafers via Surface-Initiated seATRP. Macromolecular Chemistry and Physics. 2017;218(11):1700106.

12. Chmielarz P, Yan J, Krys P, Wang Y, Wang Z, Bockstaller MR, et al. Synthesis of Nanoparticle Copolymer Brushes via SurfaceInitiated seATRP. Macromolecules. 2017;50(11):4151-4159.

13. Matyjaszewski K, Miller PJ, Shukla N, Immaraporn B, Gelman A, Luokala BB, et al. Polymers at Interfaces: Using Atom Transfer Radical Polymerization in the Controlled Growth of Homopolymers and Block Copolymers from Silicon Surfaces in the Absence of Untethered Sacrificial Initiator. Macromolecules. 1999;32(26):8716-8724.

14. Morits M, McKee JR, Majoinen J, Malho JM, Houbenov N, Seitsonen J, et al. Polymer Brushes on Cellulose Nanofibers: Modification, SI-ATRP, and Unexpected Degradation Processes. ACS Sustainable Chemistry and Engineering. 2017;5(9):76427650.

15. Khani MM, Abbas ZM, Benicewicz BC. Well-defined polyisoprenegrafted silica nanoparticles via the RAFT process. Journal of Polymer Science, Part A: Polymer Chemistry. 2017;55(9):14931501.

16. Li C, Benicewicz BC. Synthesis of Well-Defined Polymer Brushes Grafted onto Silica Nanoparticles via Surface Reversible Addition Fragmentation Chain Transfer Polymerization. Macromolecules. 2005;38(14):5929-5936.
17. Prucker O, Rühe J. Mechanism of Radical Chain Polymerizations Initiated by Azo Compounds Covalently Bound to the Surface of Spherical Particles. Macromolecules. 1998;31(3):602-613.

18. Bielawski CW, Grubbs RH. Highly Efficient Ring-Opening Metathesis Polymerization (ROMP) Using New Ruthenium Catalysts Containing N-Heterocyclic Carbene Ligands. Angewandte Chemie International Edition. 2000;39(16):29032906.

19. Jordi MA, Seery TAP. Quantitative Determination of the Chemical Composition of Silica-Poly(norbornene) Nanocomposites. Journal of the American Chemical Society. 2005;127(12):4416-4422.

20. Lynn DM, Kanaoka S, Grubbs RH. Living Ring-Opening Metathesis Polymerization in Aqueous Media Catalyzed by Well-Defined Ruthenium Carbene Complexes. Journal of the American Chemical Society. 1996;118(4):784-790.

21. Penaloza DP Jr, Sandberg DJ, Giotto MV, Seery TAP. An exfoliated clay-poly(norbornene) nanocomposite prepared by metal-mediated surface-initiated polymerization. Polymer Engineering \& Science. 2015;55(10):2349-2354.

22. Yoon PJ, Hunter DL, Paul DR. Polycarbonate nanocomposites. Part 1. Effect of organoclay structure on morphology and properties. Polymer. 2003;44(18):5323-5339.

23. Yoon PJ, Hunter DL, Paul DR. Polycarbonate nanocomposites: Part 2. Degradation and color formation. Polymer. 2003;44(18):53415354 .

24. Nevalainen K, Vuorinen J, Villman V, Suihkonen R, Järvelä $\mathrm{P}$, Sundelin J, et al. Characterization of twin-screw-extrudercompounded polycarbonate nanoclay composites. Polymer Engineering \& Science. 2009;49(4):631-640.

25. Feyz E, Jahani Y, Esfandeh M. Effect of a nanoclay/triphenyl phosphate hybrid system on the fire retardancy of polycarbonate/ acrylonitrile-butadiene-styrene blend. Journal of Applied Polymer Science. 2011;120(6):3435-3442.

26. Yoo Y, Choi KY, Lee JH. Polycarbonate/Montmorillonite Nanocomposites Prepared by Microwave-Aided Solid State Polymerization. Macromolecular Chemistry and Physics. 2004;205(14):1863-1868.

27. Carrión FJ, Arribas A, Bermúdez MD, Guillamon A. Physical and tribological properties of a new polycarbonate-organoclay nanocomposite. European Polymer Journal. 2008;44(4):968977.

28. Xiao P, Xiao M, Gong K. Preparation of exfoliated graphite/ polystyrene composite by polymerization-filling technique. Polymer. 2001;42(11):4813-4816.

29. Bansal A, Yang H, Li C, Benicewicz BC, Kumar SK, Schadler LS. Controlling the thermomechanical properties of polymer nanocomposites by tailoring the polymer-particle interface. Journal of Polymer Science Part B: Polymer Physics. 2006;44(20):2944-2950.

30. Bandi S, Schiraldi DA. Glass Transition Behavior of Clay Aerogel/Poly(vinyl alcohol) Composites. Macromolecules. 2006;39(19):6537-6545.

31. Baughman TW, Wagener KB. Recent advances in ADMET polymerization. In: Buchmeiser MR, ed. Metathesis Polymerization. Berlin Heidelberg: Springer; 2005. p. 1-42. 